\title{
An Overview Of The Motional Stark Effect Diagnostic On DIII-D And Design Work For An ITER MSE
}

C. T. Holcomb, S. L. Allen, M. A. Makowski, R. J. Jayakumar, M. F. Gu, S. Lerner, K. L. Morris, J. Latkowski, J. M. Moller, W. Meyer, R. Ellis, R. Geer, D. Behne, R. Chipman, P. Smith, S. McClain

September 20, 2007

International Workshop on Burning Plasma Diagnostics Varenna, Italy September 24, 2007 through September 28, 2007 
This document was prepared as an account of work sponsored by an agency of the United States government. Neither the United States government nor Lawrence Livermore National Security, LLC, nor any of their employees makes any warranty, expressed or implied, or assumes any legal liability or responsibility for the accuracy, completeness, or usefulness of any information, apparatus, product, or process disclosed, or represents that its use would not infringe privately owned rights. Reference herein to any specific commercial product, process, or service by trade name, trademark, manufacturer, or otherwise does not necessarily constitute or imply its endorsement, recommendation, or favoring by the United States government or Lawrence Livermore National Security, LLC. The views and opinions of authors expressed herein do not necessarily state or reflect those of the United States government or Lawrence Livermore National Security, LLC, and shall not be used for advertising or product endorsement purposes. 


\title{
An Overview Of The Motional Stark Effect Diagnostic On DIII-D And Design Work For An ITER MSE
}

\author{
C.T. Holcomb. S.L. Allen, M.A. Makowski, R.J. Jayakumar, M.F. Gu, S. \\ Lerner, K.L. Morris, J. Latkowski, J.M. Moller, W. Meyer, R. Ellis, R. \\ Geer, D. Behne, R. Chipman*, P. Smith*, S. McClain*
}

Lawrence Livermore National Laboratory, 7000 East Avenue, Livermore, California, U.S.A. 94550 ${ }^{*}$ College of Optical Sciences, The University of Arizona, 1630 East University Boulevard, Tucson, Arizona, U.S.A. 85721,

\begin{abstract}
The advanced tokamak research program at DIII-D relies critically on the measurement of the current density profile. This was made possible by the development of a Motional Stark Effect (MSE) polarimeter that was first installed in 1992. Three major upgrades have since occurred, and improvements in our understanding of critical performance issues and calibration techniques are ongoing. In parallel with these improvements, we have drawn on our DIII-D experience to begin studies and design work for MSE on burning plasmas and ITER. This paper first reviews how Motional Stark Effect polarimetry (MSE) is used to determine the tokamak current profile. It uses the DIII-D MSE system as an example, and shows results from the latest upgrade that incorporates an array of channels from a new counter-Ip injected neutral beam. The various calibration techniques presently used are reviewed. High-leverage or unresolved issues affecting MSE performance and reliability in ITER are discussed. Next, we show a four-mirror collection optics design for the two ITER MSE views. Finally, we discuss measurements of the polarization properties of a few candidate mirrors for the ITER MSE. Work supported by the U.S. Department of Energy under W-7405-ENG-48.
\end{abstract}

Keywords: Motional Stark Effect, optical diagnostic, current profile, calibration, mirrors, ITER. PACS: 52.55.Fa, 52.70.Ds, 52.70.Kz,

\section{INTRODUCTION}

Motional Stark Effect polarimetry [1,2] is a standard measurement on the DIII-D tokamak that is used to constrain equilibrium reconstruction of the current profile. The use of MSE is anticipated on ITER as well. We are applying the experience gained making MSE successful on DIII-D to the new design challenges posed by an ITERMSE. This paper is meant to provide a snapshot overview of the present state of our work on MSE for both experiments.

Briefly, MSE works by measuring the linear polarization state of a Stark component of $\mathrm{D} \alpha$ emission from a neutral beam. The $\sigma$ components are polarized perpendicular to the Stark electric field $(\mathbf{v x B})$ and the $\pi$ components are parallel. A background plasma radial electric field may also contribute to the polarization state, and this must be accounted for. The DIII-D MSE has 65 channels viewing 2 neutral 
beams from 5 arrays. Each view has either zero or one fold mirror in vacuum, followed by a vacuum window, refractive collection optics, and then the polarimeter. This consists of a dual photoelastic modulator made by Hinds Instruments and a fixed linear polarizer. This arrangement transfers the input polarization information by amplitude modulation in fiber optics to a remote lab where the signals are recovered using lock-in amplifiers or Fourier analysis. On DIII-D, the $\sigma$ component is selected using narrowband filters. The five independent views allow evaluation of the $\mathrm{Er}$ component of the measurement with good spatial resolution $(\sim 1 / 2 \mathrm{~cm}$ in the edge, $\sim 7$ $\mathrm{cm}$ in the core).

MSE measurements are combined with magnetic measurements at the wall to do realtime equilibrium fitting using the EFIT code [3] for feedback with the plasma control system [4]. The EFITs also are used for between shot analysis to guide the experiment. While kinetic data is usually incorporated into fits for further analysis, experience has shown that the MSE and magnetics only EFITs are sufficient for constraining a wide variety of current profiles. These range from simple monotonic $\mathrm{q}$ profiles with qmin $\leq 1$, to advanced tokamak discharges with qmin $\geq 2$ and negative central shear varying from weak to strong (i.e. $q 0 \approx q \min$ to $q 0>>q m i n$ ). The resulting MSE EFIT q profiles are frequently compared to independent measurements of MHD activity, and are observed to match theoretical expectations, such as, for example, tearing modes located at mode rational surfaces.

\section{Calibration and Reliability Issues For MSE}

An ideal MSE diagnostic would employee collection optics that do not alter or distort the incoming linear polarization signal. In the language of Stokes vectors and Mueller matrices that describes partially polarized light, the Mueller matrix of such a system would be identity. This is never realized in practice, because one or more fold mirrors may be required to collect light around corners and refractive elements (vacuum window, lenses, photoelastic modulator) may be susceptible to Faraday rotation in the strong magnetic field. These items will change the signal polarization and should be minimized where possible, and calibrated out where they cannot. Changes to component surface coatings due to interaction with the plasma (erosion, deposition, thermal fatigue and cracking, neutron damage and darkening, etc.) require periodic recalibration.

On the DIII-D MSE, we have made design choices that attempt to minimize the starting polarization distortion. These include using at most one steering mirror with a custom dielectric coating that maintains the incident polarization to within a few percent, and using zero Verdet constant glass for lenses to minimize Faraday rotation.

We calibrate in situ a fitting function based on Mueller matrix modeling of the collection optics [5]. The constants in the function are determined by scanning a linear polarizer placed in the vessel at the neutral beam location during a vent. Another constant that accounts for Faraday rotation is determined using fixed, thin polarizers mounted in-vessel while the vacuum magnetic field is pulsed. Finally, we are investigating a technique that uses a fixed linear polarizer followed by a rotating quarter wave plate to inject time dependent elliptical polarization into the optical train. 
Fourier analysis of the resulting signals is used to determine the system response to elliptical polarization.

In ITER, the neutron shielding requirement mandates more than one mirror in each system, and the first one or two mirrors may have to be metal rather than dielectric, which will have less ideal polarization preserving properties. Furthermore, the evolution of the front mirror calibration due to plasma interaction is expected to be far more rapid than in present experiments [6]. These create a need for a dependable calibration method that may be performed daily, or between shots. Worker vessel entry will be prohibited or extremely limited, so the calibration techniques used on DIII-D that require placing polarization references inside the tokamak will have to be done by remote insertion or by building these into the port plug. A better option that may allow frequent calibration is to fill the vessel with gas and fire the beam with known vacuum fields. The "beam-into-gas" calibration technique is presently limited to densities too low to produce statistically populated sublevels, so the resulting Stark spectrum is known to have different intensity and polarization properties than in the plasma case [7]. A full understanding of this spectrum is required before beam-intogas calibration will be reliable.

\section{ITER MSE Design Work and Mirror Studies}

We have designed a collection optics train for the edge and core ITER MSE views. Each system uses four mirrors and a single low Verdet (SFL6) lens to bring light radially out of the port plug. Figure 1a shows the edge design in a Pro |E CAD model. The core design (not shown) uses the same design form, but the first two mirrors are set farther back in the port plug, whereas the edge design has these inside the blanket shield module. The design form is the result of trade offs between desired image quality, low polarization aberration, neutron shielding, and cost. We anticipate that the first two mirrors in the edge view will have to be metal because of their proximity to the plasma. For this reason the angle of incidence on these mirrors is kept high. The other mirrors may use dielectric coatings, and their design was chosen to satisfy neutron shielding. Compared to a preliminary MSE optics design [8], the present design has: two less mirrors, about a factor of 3 less polarization aberration, about a factor of three less projected cost due to smaller number and size of mirrors, a factor of ten improvement in the image resolution, and roughly equivalent light throughput and neutron shielding. The mirror and lens diameters $(\mathrm{mm}) /$ shapes for the edge design are as follows (in order from M1 to L1): 205/spherical, 230/cylindrical, 420/spherical, 250/anamorphic, 200/spherical. For the core design, they are: 270/anamorphic, 200/spherical, 250/spherical, 250/anamorphic, 200/conic.

Polarization ray tracing was part of the design optimization, and was carried out using Jones vectors with the Zemax code. For this analysis, we assume the first two mirrors have a uniform aluminum coating, and only these have any polarization aberration. In this case, a uniform input $45^{\circ}$ linear polarization exits the system with some nonuniformity and finite ellipticity caused by a relative phase shift between $\mathrm{S}$ and $\mathrm{P}$ polarization of 2.5 to 3.2 degrees, but the pupil-averaged output angle is only rotated by $0.1^{\circ}$ to $0.2^{\circ}$. 

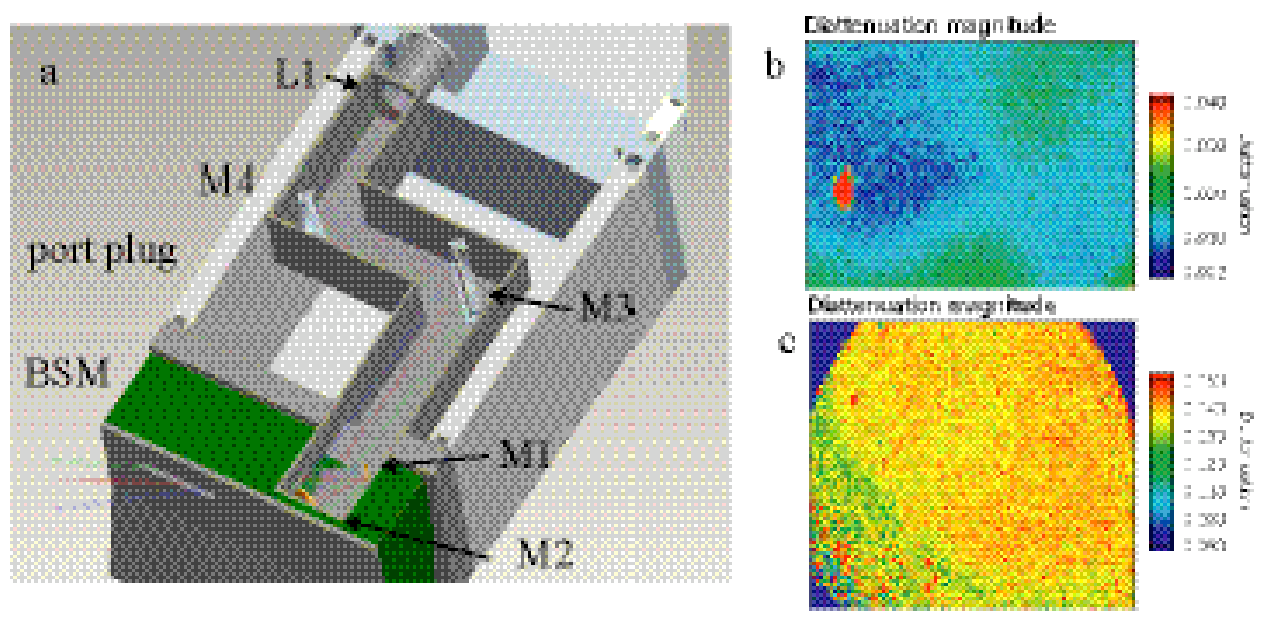

FIGURE 1. a) The LLNL $4 \mathrm{~b}$ design, b) and c) Diattenuation images for dielectric and Rh mirrors

The next step in the design validation is to measure Mueller matrices of various first mirror surface materials as a function of wavelength and angle of incidence, and use these in a more complete polarization ray trace in Code $\mathrm{V}$. These measurements will be made by a Mueller matrix angle of incidence imaging polarimeter [9]. Two example mirror candidates have already been checked using this technique for a limited number of angles. Figure $1 \mathrm{~b}$ and $\mathrm{c}$ compares the diattenuation derived from Mueller images of a custom dielectric and a Rhodium mirror. These were both performed at the same wavelength $(660 \mathrm{~nm})$ and angle of incidence $\left(60^{\circ}\right)$, and it is clear that for this case Rhodium has significantly more polarization aberration than a dielectric mirror.

\section{ACKNOWLEDGMENTS}

This work was performed under the auspices of the U.S. Department of Energy under Contract W-7405-Eng-48.

\section{REFERENCES}

1. F.M. Levinton, et al. Phys. Rev. Lett. 63, 2060 (1989).

2. D. Wroblewski et al., Rev. Sci. Instrum. 61, 3552, (1990).

3 L. L. Lao et al., Nucl. Fusion 30, 1035 (1990).

4. J.R. Ferron et al., Nucl. Fusion 38, 1055 (1998).

5. M.A. Makowsi et al., Rev. Sci. Instrum. 76, 023706 (2005).

6. "ITER MSE Diagnostic First Mirror Analysis", J.P. Allain, J.N. Brooks, A. Hassanein, Argonne National Laboratory, presented by Dave Johnson, USBPO Diagnostics Task Group Meeting, General Atomics, San Diego, CA, February 2007.

7. M.F. Gu et al., "Atomic Models for Motional Stark Effect Diagnostics", submitted to J. Phys. B: At. Mol. Opt. Phys.

8. A. Malaquias et al., Rev. Sci. Instrum. 75, 3393 (2004).

9. J. Pezzaniti and R.A. Chipman, Optical Engineering 34, 1558, (1995).

This work was performed under the auspices of the U.S. Department of Energy by UC, Lawrence Livermore National Laboratory under Contract W-7405-Eng-48. 